\title{
All-day radiative cooling using beam-controlled architectures
}

\author{
Lyu Zhou ${ }^{1}$, Haomin Song ${ }^{1}$, Jianwei Liang ${ }^{2}$, Matthew Singer ${ }^{1}$, Ming Zhou ${ }^{3}$, Edgars Stegenburgs ${ }^{2}$, \\ Nan Zhang ${ }^{1}$, Tien Khee $\mathbf{N g}^{2}$, Zongfu $\mathbf{Y u}^{3}$, Boon Ooi ${ }^{2}$, Qiaoqiang Gan ${ }^{1}$ \\ ${ }^{1}$ Department of Electrical Engineering, The State University of New York at Buffalo, Buffalo, NY 14260 \\ ${ }^{2}$ KAUST Nanophotonics Lab, King Abdullah University of Science and Technology, Thuwal 23955-6900, 8 Saudi Arabia \\ ${ }^{3}$ Department of Electrical and Computer Engineering, University of Wisconsin, Madison, Wisconsin 10 53705, USA \\ Email:qqgan@buffalo.edu
}

\begin{abstract}
We report an inexpensive planar polydimethylsiloxane (PDMS)/metal thermal emitter in a beam-controlled architecture for all-day radiative cooling and realized $\sim 11^{\circ} \mathrm{C}$ reduction compared with the ambient temperature. (c) 2019 The Author(s)
\end{abstract}

OCIS codes: 350.5610 Radiation; 010.1320 Atmospheric transmittance

Air conditioning is a significant energy consumer and a major driver of global peak electricity demand. For instance, air conditioning consumes $\sim 15 \%$ of the primary energy used by buildings in the United States [1]. Therefore, a passive cooling strategy that cools without any electrical input could have significant impact on global energy consumption. In recent years, an ancient cooling technology was brought onto the stage due to its non-energy consuming characteristic. The so-called technology, radiative cooling, has been exploited for decades. However, since the solar heating will dominate during the daytime, it is of great challenge to achieve efficient radiative cooling during daytime, when peak cooling demand actually occurs. In 2014, researchers at Stanford University first reported a photonic structure that can function well during both daytime and nighttime [2]. Several strategies were also reported followed by this pioneering work $[3,4]$. In this report, we propose and experimentally demonstrate a PDMS/metal thermal emitter for all-day radiative cooling. A spectral-selective architecture is also implemented to suppress the solar input and taper the thermal radiation. Outdoor experiments were performed in Buffalo, NY, realizing an average $6{ }^{\circ} \mathrm{C}$ temperature reduction through 48 hours.

A

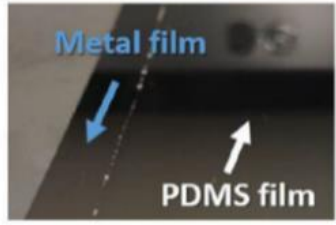

C

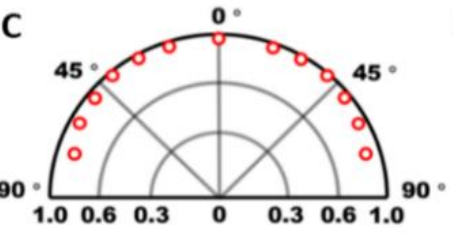

B

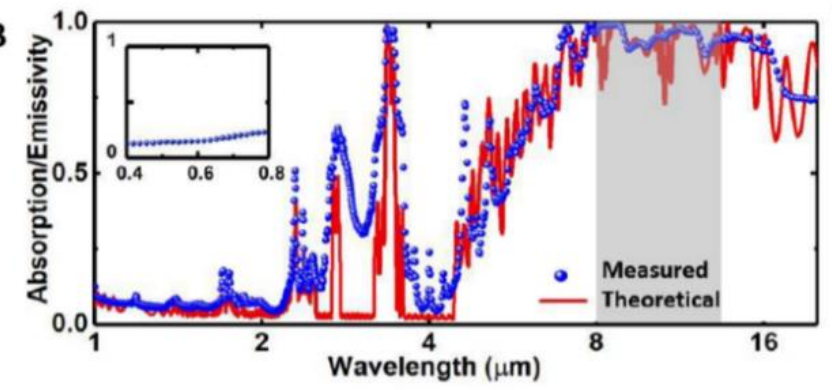

D
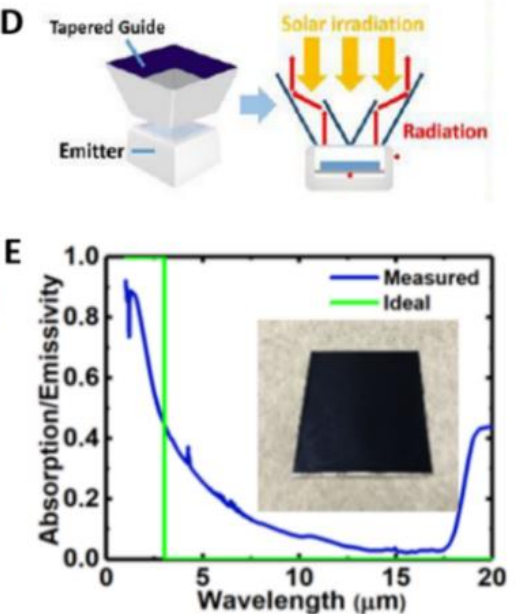

Fig. 1 (A) The photo of the planar emitter; (B) The measured (blue dot) and the modeled (red line) absorption spectrum of the planar emitter; (C) The measured angular absorption of planar emitter at $10 \mathrm{~m}$; (D) The schematic of the beaming structure; (E) The absorption spectrum of the selective material.

The Earth's atmosphere has a transparent window for electromagnetic (EM) waves between 8-13 $\mu \mathrm{m}$, corresponding to the peak thermal radiation spectral range of terrestrial objects at typical ambient temperature (e.g. $\sim 20^{\circ} \mathrm{C}$ to $45^{\circ} \mathrm{C}$ ). Through this window, thermal energy can be transferred from the thermal body to cold outer space. On the other hand, significant energy is brought onto the earth surface by the solar illumination. To achieve daytime radiative cooling, it is essential to engineer the absorption spectrum of the emitter. In this article, we report a planar PDMS/metal thermal emitter that is useful for efficient radiative cooling over large areas. As illustrated in Fig. 1A, the emitter is composed of a 150- m-thick PDMS film on aluminum plate. Both measured and modeled absorption spectrum domain is given in Fig. 1B, which exhibit strong absorption in mid-infrared wavelength range and weak absorption in visible-to-near infrared wavelength range. In addition, to reveal the angular-dependence of the absorption, the absorption at $10 \mathrm{~m}$ was also measured, as shown in Fig. 1C. As one can see, the absorption/emissivity is approximately omnidirectional. 
Thus, it is a technical challenge to collect the thermal radiation efficiently to optimize the radiative cooling performance. Beam control of the thermal emission is therefore of interest to address this limitation. To address this issue, we introduce a spectral-selective architecture in the cooling system, as shown in Fig. 1D. The absorption spectrum of the selective absorbing material is given in Fig. 1E, showing a high absorption in visible-to-near infrared wavelength range and weak absorption in mid infrared wavelength range. Ideally, such tapered structure can block all downwards solar irradiation while reflect the thermal emission from the thermal emitter. Moreover, due to the geometric design of the structure, the output radiation can be confined within a certain angle range, showing a better tolerance to the complicated environment. By employing this spectral-selective architecture, it is possible to implement radiative cooling systems in a very complicated environment, for example the urban area where the demand for cooling is mostly significant. Such a discussion has never been made before, since most of the groups are focused on optimizing the material. Next, we will experimentally demonstrate the beaming effect on radiative cooling.
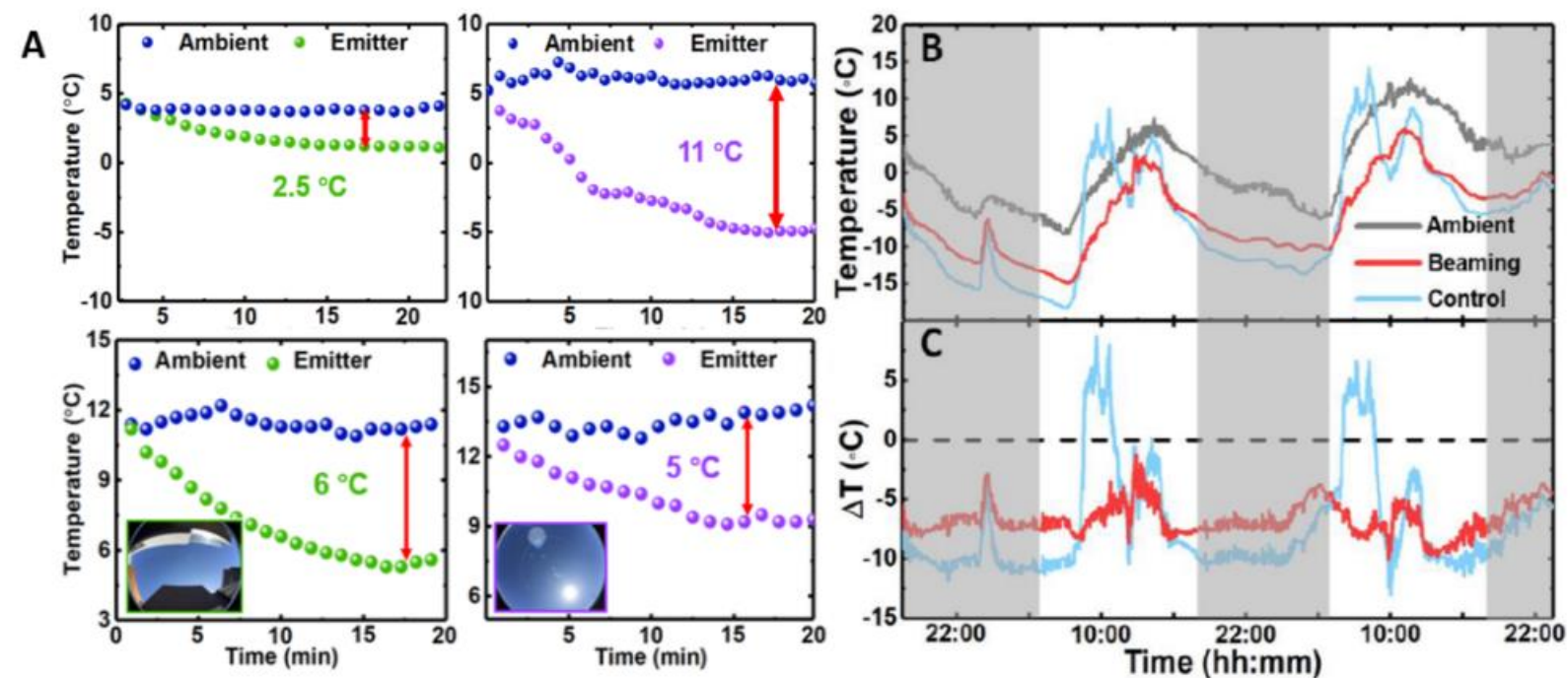

Fig. 2 The outdoor cooling test in Buffalo. (A) The cooling at two different locations. The tests were performed without beaming structure (upper panel) and with beaming structure (lower panel). (B) The 48-hours continuous cooling test. (C) The corresponding temperature reduction in $(\mathrm{B})$.

To illustrate the critical impact of the environment, we measured the temperature reduction of two groups at two different locations in Buffalo, respectively. The test locations' photos are taken by the fisheye camera and shown in the inset of Fig. 2A, demonstrating the access to clear sky. The temperature was measured in the system that with and without the beaming structure. As one can see, for the group without the beaming structure, the emitter's temperature reduction varied from $2.5^{\circ} \mathrm{C}$ to $11^{\circ} \mathrm{C}$ as the test environment changed. Meanwhile, for the group with the beaming structure, the temperature reduction was reasonable steady. These results show that the beaming structure could efficiently eliminate the environmental effects. The weaker performance in open was caused by the imperfect optical characteristic of the selective material, where further optimization is under investigation but beyond the scope of this work. At last, a 48-hours cooling test was performed in a typical America neighborhood, as shown in Fig. 2B-C. Remarkably, the temperature of the beaming structure was always lower than ambient temperature, while the temperature of the control group will increase dramatically above the ambient temperature when exposed to sun. In general, the beaming group can achieve an average $6{ }^{\circ} \mathrm{C}$ temperature reduction through the day, which corresponds to $\sim 80 \mathrm{~W} / \mathrm{m}^{2}$ cooling power.

In summary, we developed a passive cooling technology by exploiting the sky as a cold source. The proposed PDMS/metal emitter is low-cost and suitable for massive production. In addition, a spectral-selective beaming structure was introduced to improve the sensitivity of the environment. All-day continuous test was performed, demonstrating an effective cooling effect even under the solar illumination. Compared to the traditional cooling technology (e.g. air conditioning), the proposed technology is totally energy-free and operates at a reasonably low cost, showing a great potential in its commercial applications.

\section{Reference}

[1] J. K. Kelso, 2011 Building energy data book. Department of Energy (2012).

[2] A. P. Raman, et al., Passive radiative cooling below ambient air temperature under direct sunlight. Nature 515, 540 (2014).

[3] Y. Zhai et al., Scalable-manufactured randomized glass-polymer hybrid metamaterial for daytime radiative cooling. Science 355, 1062 (2017).

[4] J.Mandal et al., Hierachically porous polymer coatings for highly efficient passive daytime radiative cooling. Science 362, 315 (2018). 